\title{
THE RELATIONSHIP BETWEEN LEFT VENTRICULAR FRACTIONAL SHORTENING AND INTRAVENOUS ADMINISTRATION OF STEM CELLS IN LABORATORY RABBITS PRESENTING CHRONIC MYOCARDIAL INFARCTION
}

\author{
IONEL CIPRIAN POP ${ }^{1}$, OVIDIU GRAD ${ }^{1}$, EMOKE PALL ${ }^{2}$, \\ COSMIN PESTEAN ${ }^{2}$, MIRCEA MIRCEAN ${ }^{2}$, ION AUREL MIRONIUC ${ }^{1}$
}

\author{
12-nd Surgical Clinic, Iuliu Hatieganu University of Medicine and Pharmacy, \\ Cluj-Napoca, Romania \\ ${ }^{2}$ Faculty of Veterinary Medicine, University of Agricultural Sciences and \\ Veterinary Medicine, Cluj-Napoca, Romania
}

\begin{abstract}
Background and aims. The present study conducted from March 2012 to July 2013 aimed to evaluate from echocardiographic point of view the effects of peripheral intravenous administration of mesenchymal stem cells (MSCs) in laboratory rabbits presenting 30 days old chronic myocardial infarction.

Material and methods. 30 days after the induction of an acute myocardial infarction in 40 laboratory rabbits by direct ligation of the left anterior descending coronary artery at about $10 \mathrm{~mm}$ from the apex, we injected $1 \times 106$ MSCs in the auricular vein in a group of 30 rabbits, and a group of 10 rabbits were used as controls. 30 days after the injection of stem cells the left ventricular (LV) fractional shortening (FS) was evaluated by echocardiography and compared with the control rabbits.

Results. In control rabbits, echocardiography revealed akinesis of apex, interventricular septum kinetics was also impaired, FS being approximately 6\%. In 80\% (24 rabbits) of the injected rabbits the FS of the LV was significantly greater than in the witness group $(26+/-2 \%$, $p<0.0001)$. At 13.3\% (4 rabbits) of the injected rabbits the FS of the LV showed no improvement in comparison with the control group $(6.5+/-1 \%)$.

Conclusion. An improvement of LV SF 30 days after MSCs were injected $(p<0.0001)$ was noted. We have to further determine if this improvement of the LV function is correlated with any histopathological changes and if it is not lost in time. Also, further studies needs to evaluate if there is any significant change in the overall mortality.
\end{abstract}

Keywords: mesenchymal stem cells, acute myocardial infarction, chronic myocardial infarction, fractional shortening, left ventricle.

\section{Background and aims}

Ischemic heart disease remain a major public health problem in western countries despite a better understanding of its pathophysiology and therapeutic managment. Cardiovascular disease is the number one cause of death worldwide $[1,2]$. Congestive heart failure (CHF) is the end stage of many heart diseases, but ischemic heart disease

Manuscript received: 28.03 .2014

Received in revised form: 13.01.2015

Accepted: 14.01.2015

Address for correspondence: doc_cipri@yahoo.com is one of the most common etiology. CHF is five times more common in those who have had an acute myocardial infarction (AMI) than in those who have not. The prognosis for those with established CHF is generally poor and worse than for those with most malignancies or AIDS, with a oneyear mortality rate as high as 40 percent and a five-year mortality between 26 and 75 percent [3]. If, in case of an AMI, the medical and interventional management are the „the gold standard”, once the chronic myocardial infarction develops, the medical management of the patient is limited 
and progression towards CHF is often certain. Cellular cardiomyoplasty may be a promising approach to improve cardiac regeneration or vascularization after chronic myocardial infarction develops [4]. In the international literature it has been reported that transplantation of fetal or neonatal cardiomyocytes can increase the thickness of the infarct wall and LV stroke volume, decrease LV end-systolic volume, and improve LV ejection fraction in a rat model of myocardial infarction $[5,6]$ and induce neoangiogenesis [7].

As sometimes only the peripheral intravenous route is available, the present study, conducted between March 2012-July 2013 at the Iuliu Hatieganu University of Medicine and Pharmacy Cluj Napoca's Biobase in collaboration with the University of Agricultural Sciences and Veterinary Medicine Cluj-Napoca, aimed at evaluating the effects of peripheral intravenous administration of stem cells 30 days after the induction of an acute myocardial infarction in laboratory rabbit. This article highlights the echocardiographic changes of LV FS at 30 days after peripheral intravenous administration of stem cells in rabbits with chronic myocardial infarction.

\section{Materials and methods}

All procedures of the experiments complied with the standards of animal care. Working protocols were approved by the Ethics Committee of the Iuliu Haţieganu University of Medicine and Pharmacy Cluj-Napoca [8].

\section{Bone marrow harvest}

In order to harvest the MSCs we used $20 \mathrm{New}$ Zealand White rabbits (3-3.5 kg) 1 year of age. Rabbits were from the Biobase of the Iuliu Hatieganu University of Medicine and Pharmacy, Cluj-Napoca. Marrow punctures were made after general and local anesthesia. Neuroleptanalgesia was performed using acepromazine and ketamine, and local anesthesia was performed with alphacain and adrenaline in separate points. Local antisepsis and asepsis were performed prior to bone puncture. For intervention the animals were restrained in lateral decubitus, at the iliac crest a blade skin incision was performed followed by sterile needle penetration in the spinal canal approximately $1 \mathrm{~cm}$ from the iliac crests. MSC acquired from bone marrow aspiration were grown on standard culture mediums $[9,10,11,12,13]$.

\section{MSCs}

\section{Model of myocardial infarction and injection of}

For this study we used 40 rabbits, breed New Zealand White (3-3.5 kg), age of 1 year. The acute myocardial infarction was induced by direct ligation of the left anterior descending (LAD) coronary artery at about 10 $\mathrm{mm}$ from the apex, followed by a period of 30 days in witch the rabbits were kept at standard conditions of temperature, humidity and food for the myocardial infarction to become chronic [14].

After the chronic myocardial infarction was achieved, 30 rabbits were injected with $1 \times 10^{6}$ stem cells/ rabbit using the auricular vein under mild sedation of Ketamine $(15 \mathrm{mg} / \mathrm{kg})[15,16,17,18]$. Another group of 10 rabbits with chronic myocardial infarction was kept as controls.

\section{Echocardiographic assessment}

30 days after the administration of the stem cells both groups were subjected to a blind echocardiographic evaluation of the LV, with special interest in FS.

In the literature the left ventricle FS is considered as: normal from $25-40 \%$; mildly impaired from $20-25 \%$, moderately impaired from $15-20 \%$; and severely impaired if $<15 \%$ [19].

In our previous study, when we developed the experimental model, the value of FS in normal rabbits was $50+/-2 \%$, value that we considered as normal for this study [14].

The ultrasound examination was performed through a subxiphoid window with rabbit shaved, sedated with Ketamine $(15 \mathrm{mg} / \mathrm{kg})$ and prone. We tried to focus on the LV, and mostly on its FS (end diastolic diameter - end systolic diameter/end diastolic diameter X 100) in MMode [20].

\section{Statistical analysis}

All data are presented as mean +/- SEM. Comparisons between groups were made by Student $t$ test or Fisher's exact test, where appropriate. Results were considered statistically significant if $\mathrm{p}<0.05$.

\section{Results}

In control rabbits, echocardiography performed 60 days after the induction of the acute myocardial infarction evidenced a severe impaired LV function, the FS being approx. 6\%, akinesia of LV apex was also noted and interventricular septum kinetics was also impaired (Fig. 1).

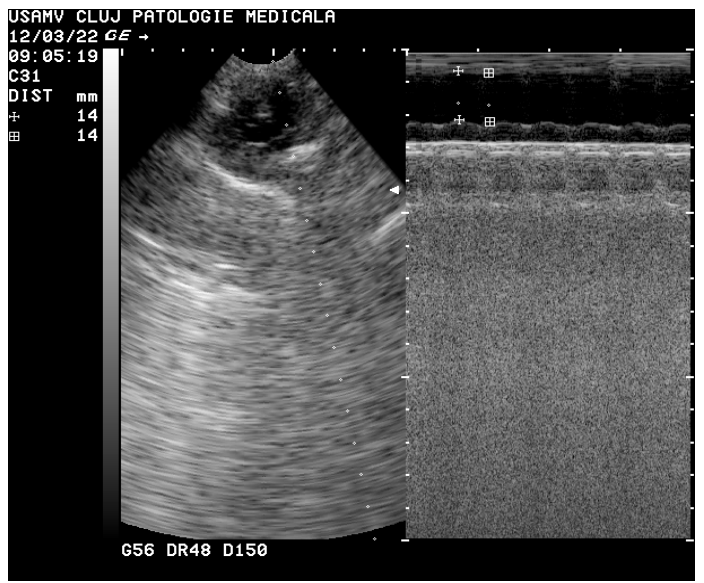

Figure 1. Echocardiography in a control rabbit showing akinesia of the apex. 

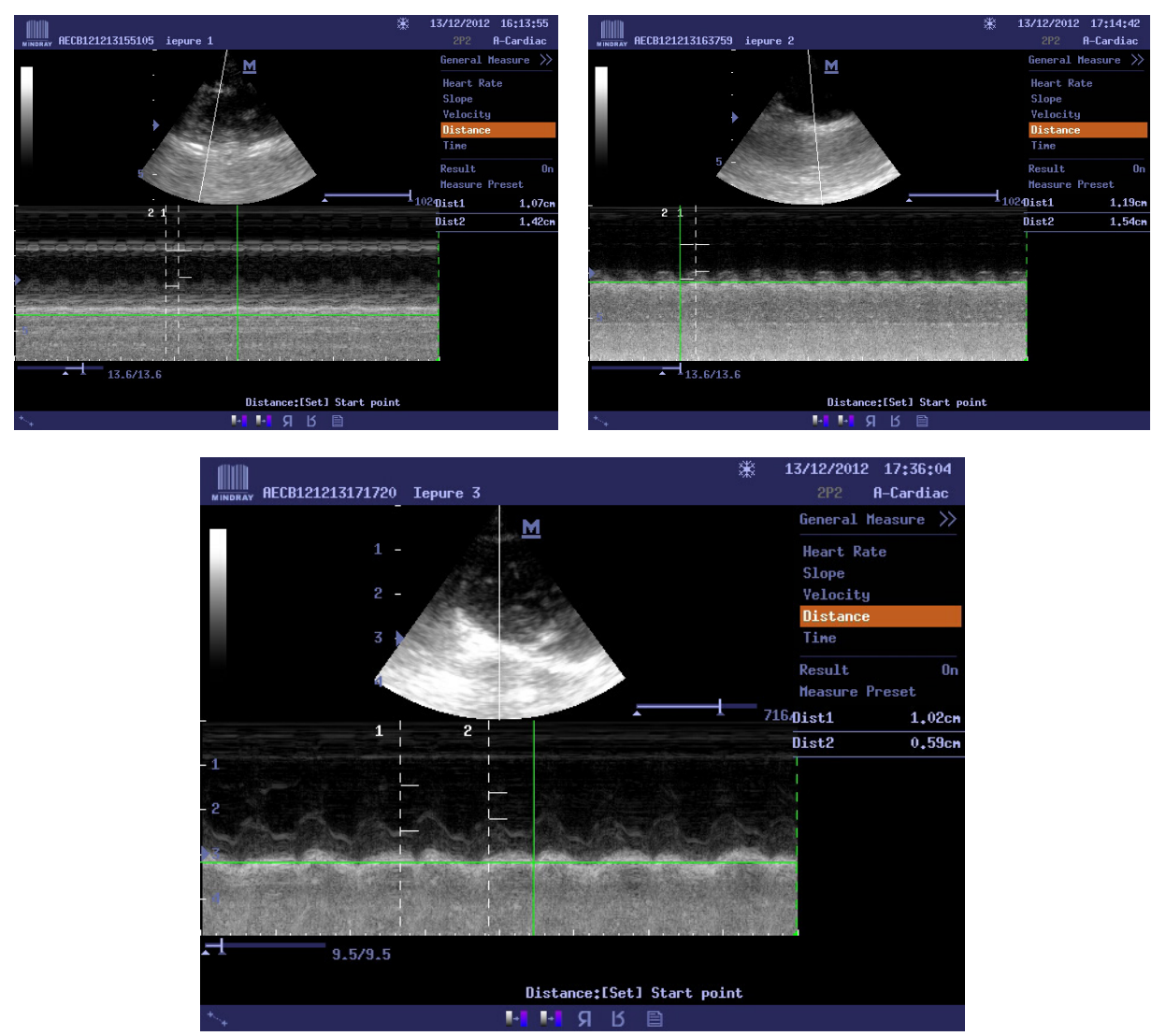

Figure 2. LV echocardiography in injected rabbits showing an average FS of $32 \%$.

Two of the injected rabbits were excluded from the study because the FS was suspiciously high $(35 \%$, respectively $36 \%$ ).

In $80 \%$ (24 rabbits) of the injected rabbits the FS of the LV was significantly greater than in the control group ( $26+/-2 \%, \mathrm{P}<0.0001)$.

In $13.3 \%$ (4 rabbits) of the injected rabbits the FS of the LV showed no improvement in comparison with the control group (6.5+/-1\%) (Fig. 2).

At this stage of the experiment we had no mortality among the rabbits, but before the experimental model was well defined the mortality reached in some lots up to $30 \%$ mostly intraoperatively or at a second exposure to anaesthetic drugs.

\section{Discussion}

Since ischemic heart disease remains a major public health problem in western countries, a continuous research for alternative therapies remains a priority. The stem cell ability to differentiate into mesoderm and non mesoderm-derived tissues has aroused the interest of many researchers to evaluate their effect in cardiovascular disease management, and has proved to be an effective therapy $[11,12,21]$.

Since most studies uses an intralesional administration of MSCs with good results, but in practice not always possible, we wanted to evaluate the impact of peripheral intravenous administration [22,23].

The FS reflects the left ventricle systolic function. If we consider the left ventricle FS as: normal from 50+/$2 \%$; and severely impaired if $<15 \%$, the results obtained are promising [14].

We admit that an echocardiography study done before and after administrating the MSCs in every rabbit would have had reduced to minimum any subjective findings in LV evaluation, but the low tolerance of general anaesthetic with high mortality rate (up to $30 \%$ ), made us abandon this strategy.

We excluded from the study the 2 rabbits with FS of $35 \%$, respectively $36 \%$, because we believe the distance of LAD ligation was probably closer to the apex that it was supposed to be (it corresponds to a FS of the rabbits who underwent LAD ligation at approximately $5 \mathrm{~mm}$ from apex at experimental model) [14].

Even if LAD artery ligation at $10 \mathrm{~mm}$ from apex caused a severe LV impairment $(100 \%$ of the control rabbits) and in the group receiving intravenous MSCs we noticed an increase of FS in most of the cases, 4 weeks after the MSCs were administered, we have to admit the limitation of the study since we realize that the LV function is best evaluated measuring its ejection fraction (which was not possible to measure due to device we had access to). We were also unable to correlate this improvement with the clinical symptomatology (very hard to evaluate on an 
animal model).

There are also questions left to be answered, like: will this improvement we noticed last? Wangde et al [24] in his experiment injected MSCs into the scar of a 1-weekold myocardial infarction in Fischer rats and an improved of global LV function 4 weeks after was obtained, but this benefit was lost 6 months later. Further more he noted that histopathologically, the LV function improvement 4 weeks after MSCs were administered was achieved without an increased thickening of the infarcted muscle wall. They concluded that the injected MSCs may had produced humoral substances that had a beneficial effect over the surrounding viable muscle cells in some way, a so-called paracrine effect. We have no reason to exclude this paracrine effect theory of the injected MSCs over the improvement of LV FS in our study, the histology will hopefully answer this question.

We also don't know at this moment the impact of pulmonary passage over the injected MSCs and if there is a relationship between this and the "unresponsive" rabbits. We haven't found a reason why $13.3 \%$ of the injected rabbits showed no improvement of LV FS in comparison with the control group.

Even if our study does no report tumor formation, question remains regarding the long term safety of using stem cells $[25,26]$.

\section{Conclusion}

An improvement (comparing with the control group) of LV SF in injected rabbits, 30 days after MSCs were administered $(\mathrm{p}<0.0001)$ was noted.

We have to further determine if this improvement of the LV function is correlated with any histopathological changes and if it is not lost in time.

Also further studies need to evaluate if there are any significant changes in the overall mortality.

We cannot say at this stage if peripheral intravenous route is or is not a viable one for the administration of MSCs for ischemic heart pathology.

\section{References:}

1. WHO (World Health Organization). The World Health Report 2002: Reducing Risks, Promoting Healthy Life. Geneva 2002b; WHO.

2. Murray C J, Lopez A D. Global Burden of Disease and Injury Series, Vols. I and II, Global Health Statistics. Harvard School of Public Health, Boston 1996;.

3. McMurray JJ, Stewart S. Epidemiology, aetiology, and prognosis of heart failure. Heart. 2000; 83:596-602.

4. Reffelmann T, Kloner RA. Cellular cardiomyoplasty: cardiomyocytes, skeletal myoblasts, or stem cells for regenerating myocardium and treatment of heart failure? Cardiovasc Res. 2003;58:358-368.

5. Müller-Ehmsen J, Peterson KL, Kedes L, Whittaker P, Dow JS, Long TI, et al. Rebuilding a damaged heart: long-term survival of transplanted neonatal rat cardiomyocytes after myocardial infarction and effect on cardiac function. Circulation. 2002; $105: 1720-1726$.

6. Yao M, Dieterle T, Hale SL, Dow JS, Kedes LH, Peterson $\mathrm{KL}$, et al. Long-term outcome of fetal cell transplantation on postinfarction ventricular remodeling and function. J Mol Cell Cardiol. 2003;35:661- 670.

7. Reffelmann T, Dow JS, Dai W, Hale SL, Simkhovich BZ, Kloner RA. Transplantation of neonatal cardiomyocytes after permanent coronary artery occlusion increases regional blood flow of infarcted myocardium. J Mol Cell Cardiol. 2003;35:607613.

8. Grad NO, Pop IC, Mironiuc IA. Stem Cell Therapy and Research. Benefits and ethical challenges Journal for the Study of Religions and Ideologies 2012;11:190-205.

9. Grad ON, Pall E, Mironiuc IA. Izolation, Culture and detection of mesenchymal stem cells in tissues for critical limb ischemia model in rat. Clujul Medical. 2013;86:65-68.

10. Zhang L, Chan C. Isolation and enrichment of rat mesenchymal stem cells (MSCs) and separation of single-colony derived MSCs. J Vis Exp. 2010;(37):1852.

11. Izadpanah R, Trygg C, Patel B, Kriedt C, Dufour J, Gimble JM, et al. Biologic properties of mesenchymal stem cells derived from bone marrow and adipose tissue. J Cell Biochem. 2006;99:12851297.

12. Williams AR, Hare JM. Mesenchymal stem cells: biology,pathophysiology, translational findings, and therapeutic implications for cardiac disease. Circ Res. 2011;109:923-940.

13. Asahara T, Murohara T, Sullivan A, Silver M, van der Zee R, $\mathrm{Li} \mathrm{T}$, et al. Isolation of putative progenitor endothelial cells for angiogenesis. Science. 1997;275:964-967.

14. Pop IC, Grad ON, Pestean C, Taulescu M, Mircean M, Mironiuc IA. Induction of a chronic myocardial infarction in the laboratory animal-experimental model. Clujul Medical 2013;86:334-339.

15. White GL, Holmes DD. A comparison of ketamine and the combination ketamine-xylazine for effective surgical anesthesia in the rabbit. Lab Anim Sci. 1976;26:804-806.

16. Correll GE, Maleki J, Gracely EJ, Muir JJ, Harbut RE. Subanesthetic ketamine infusion therapy: a retrospective analysis of a novel therapeutic approach to complex regional pain syndrome. Pain Med. 2004; 5: 263-275.

17. Fujikawa DG. Neuroprotective effect of ketamine administered after status epilepticus onset. Epilepsia. 1995;36(2):186-195.

18. Fontes-Sousa AP, Moura C, Carneiro CS, Teixeira-Pinto A, Areias JC, Leite-Moreira AF. Echocardiographic evaluation including tissue Doppler imaging in New Zealand white rabbits sedated with ketamine and midazolam. Vet J. 2009;181:326-331. 19. Lang RM, Bierig M, Devereux RB, Flachskampf FA, Foster E, Pellikka PA, et al. Recommendation for chamber quantification. Eur J Echocardiogr. 2006;7:79-108.

20. Douglas PS, Garcia MJ, Haines DE, et al. ACCF/ASE/AHA/ ASNC/HFSA/HRS/SCAI/SCCM/SCCT/SCMR 2011 Appropriate Use Criteria for Echocardiography. A Report of the American College of Cardiology Foundation Appropriate Use Criteria Task Force, American Society of Echocardiography, American Heart Association, American Society of Nuclear Cardiology, Heart Failure Society of America, Heart Rhythm Society, Society for Cardiovascular Angiography and Interventions, Society of Critical Care Medicine, Society of Cardiovascular Computed Tomography, and Society for Cardiovascular Magnetic Resonance Endorsed by the American College of Chest Physicians. J Am 
Coll Cardiol 2011;57:1126-1166.

21. Segers VF, Lee RT. Stem-cell therapy for cardiac disease. Nature. 2008;451:937-942.

22. Asahara T, Masuda H, Takahashi T, Kalka C, Pastore C, Silver $\mathrm{M}$, et al. Bone marrow origin of endothelial progenitor cells responsible for postnatal vasculogenesis in physiological and pathological neovascularization. Circ Res. 1999;85:221-228.

23. Pittenger MF, Mackay AM, Beck SC, Jaiswal RK, Douglas $\mathrm{R}$, Mosca JD, et al. Multilineage potential of adult human mesenchymal stem cells. Science. 1999;284:143-147.

24. Dai W, Hale SL, Martin BJ, Kuang J-Q, Dow JS, Wold
LE, et al. Allogeneic mesenchymal stem cell transplantation in postinfarcted rat myocardium: short- and long-term effects. Circulation 2005;112:214-223.

25. Jeong JO, Han JW, Kim JM, et al. Malignant tumor formation after transplantation of short-term cultured bone marrow mesenchymal stem cells in experimental myocardial infarctin and diabetic neuropathy. Circ Res, 2011;108:1340-1347.

26. Paunescu V, Bojin FM, Tatu CA, Gavriliuc OI, Rosca A, Gruia AT, et al. Tumour-associated fibroblasts and mesenchymal stem cells: more similarities than differences. J Cell Molec Med. 2011;15:635-646. 